\title{
Cambio climático, inundaciones y "lagunas" de información. Análisis de inundaciones a través del rastreo de artículos periodísticos en el Gran La Plata (Buenos Aires, Argentina)
}

\author{
Climate change, flooding and information "lagoons". \\ Analysis of floods through the tracking of newspaper articles \\ in the Greater La Plata region (Buenos Aires, Argentina) \\ Daniela Vanesa Rotger[l] \\ María Aversa ${ }^{[I]}$ \\ Estefanía Jáuregui[ ${ }^{[11]}$
}

Resumen

El escrito incorpora una metodología para el registro de inundaciones en la región Gran La Plata a partir del relevamiento en un medio periodístico local (diario El Día) en un período que abarca desde 1911 a 2014. Los principales resultados refieren al análisis de las inundaciones según tipo de evento de precipitación, las localidades afectadas y el reconocimiento de los años críticos. Esta metodología podría dar lugar a nuevas líneas de investigación y plataformas colaborativas, como aplicaciones que sirvan para alertas tempranas, prevención y mitigación; estrategias orientadas a reconocer la percepción social del riesgo.

Palabras clave: cambio climático; inundaciones; información; artículos periodísticos.

\begin{abstract}
The document incorporates a methodology for recording floods in the Greater La Plata region based on a survey in a local newspaper (EI Día) covering the period from 1911 to 2014. The main results refer to an analysis of floods according to the type of precipitation event, the affected localities, and a recognition of the critical years. This methodology can lead to new lines of research and collaborative platforms, such as applications for early warning, prevention and mitigation, and to strategies oriented to recognize the social perception of risk.
\end{abstract}

Keywords: climate change; flooding; information; newspaper articles. 


\section{Introduccion}

La microrregión denominada como Gran La Plata (en adelante GLP) es un conglomerado urbano situado sobre el borde costero sur del Río de La Plata, integrado por los partidos de La Plata, Berisso y Ensenada. En este conglomerado las inundaciones son un suceso periódico que deriva de dos fenómenos: las precipitaciones por encima de la media, que producen el desborde de los arroyos que atraviesan la zona urbana y el fenómeno conocido como sudestada, que eleva el nivel del Río de la Plata e inunda la zona litoral.

Históricamente ambos fenómenos han representado las amenazas más importantes de la región, sin embargo la sudestada siendo inherente a la situación litoral, ha tenido un desarrollo constante desde los orígenes del conglomerado, por lo que se configuró como una amenaza recurrente, para la que tanto la población como las instituciones, han ido construyendo resiliencia. Sin embargo, los episodios de precipitaciones de gran magnitud, si bien se registran desde principios de siglo XX, han incrementado su recurrencia e intensidad en los últimos años. Entre los principales episodios se destaca la precipitación acontecida el 2 de abril de 2013, en la que se registró una pluviometría de $300 \mathrm{~mm}$ en $2 \mathrm{hs}$ y 392 en $24 \mathrm{hs}$, ocasionando el anegamiento de unas 3500 ha (Facultad de Ingeniería, 2013), lo que derivó en graves daños materiales, y la pérdida de 89 vidas humanas.

La gravedad del evento de abril de 2013, ha impulsado estudios interdisciplinarios acerca del riesgo en el GLP, las amenazas y los distintos tipos y grados de vulnerabilidad, como primer paso para avanzar en la gestión colectiva del riesgo, concepto en el que se enmarca el presente artículo. Para desarrollar esta noción y comprender el objetivo del trabajo, así como del proyecto marco ${ }^{1}$ en el que se sitúa, es necesario referir brevemente al marco conceptual de la gestión del riesgo.

Se entiende a la noción de riesgo como la probabilidad de que a una población le ocurra algo nocivo (Lavell en Fernández, 1996). Su existencia está supeditada a la convergencia de una amenaza o peligro y una población vulnerable a su impacto. La amenaza se define como "la posible ocurrencia de fenómenos intensos y nocivos" (Cardona, 2001, p. 1). Representa al fenómeno natural que se suscita para que exista una situación de riesgo, como lo es en el caso de estudio un evento de precipitación, al riesgo de inundación. Lavell (2001) y Pérez (2013) coinciden en que la vulnerabilidad como factor concomitante a la amenaza, refiere a la percepción y susceptibilidad al daño, considerando que la propensión a sufrir daños es diferencial según grupos sociales. El carácter relativo de la vulnerabilidad permite abordarla desde una doble lectura: como la susceptibilidad de una sociedad ante la exposición a una amenaza; y como la capacidad que tiene una sociedad de afrontarla (Rotger y Sanz Ressel, 2016). En cuanto a los tipos de vulnerabilidad, históricamente estas fueron abordadas con un fuerte énfasis en los aspectos físicos del territorio, enfoque vinculado al predominio de la ingeniería y la geología en el abordaje inicial de los desastres (Ribera Masgrau, 2004). Este enfoque tecnocrático sigue orientado hacia el estudio de la amenaza como detonante del desastre y no a los factores que favorecen su existencia, es decir a la vulnerabilidad social, 
que permanece en el tiempo y es producto del nivel socioeconómico, siendo un factor determinante en los países en desarrollo (Cardona, 1993).

La revisión crítica en cuanto a la manera de abordar los desastres ha ampliado los horizontes conceptuales en torno a la noción de vulnerabilidad (Lavell en Fernández, 1996), sin embargo no basta con el conocimiento de los expertos para conocer la vulnerabilidad y el riesgo real; para esto es necesario investigar las representaciones de las comunidades afectadas, evitando un "divorcio entre el imaginario formal que sustenta las intervenciones externas y los diversos imaginarios locales pero reales que maneja la población" (Maskrey ,1997, p. 5).

Cardona (2001) apunta a la necesidad de diferenciar las lecturas acerca de la vulnerabilidad que pueden hacer ingenieros, hidrólogos, etc. de la representación que las personas tienen, razón por la que hoy se justifica la necesidad de profundizar acerca de la percepción social del riesgo, tanto desde el punto de vista individual como colectivo, aspecto fundamental para poder encontrar mecanismos de reducción de los desastres.

Según el mismo autor la gestión colectiva del riesgo involucra tres tipos de políticas públicas: identificación del riesgo (percepción individual, representación social y estimación de carácter objetivo), reducción del riesgo (prevención y mitigación) y manejo de desastres (respuesta y recuperación). El autor incorpora una cuarta política que es la transferencia del riesgo, con escaso desarrollo (Cardona, 2001).

Como parte de un grupo de trabajo interdisciplinar cuyo objetivo ha sido la construcción de una matriz del riesgo en la microrregión; se estructuró un equipo ${ }^{2}$ orientado a formalizar un registro histórico de inundaciones en una escala que integrara los tres partidos, generando así un insumo para la posterior construcción de la matriz antes mencionada. La metodología presentada articula la vulnerabilidad y la percepción del riesgo, constituyéndose en una herramienta que alberga una gran potencialidad en la construcción social del riesgo como camino para la prevención y mitigación de daños ex post al evento, mediante la constitución de políticas púbicas de tipo participativo, avanzando en la transferencia del riesgo, un tema muy poco desarrollado en la microrregión.

Este trabajo resulta de interés, dado que el análisis en relación a estos temas ha revelado que el estudio de territorios vulnerables vinculados al ordenamiento del territorio y el ambiente a escala metropolitana, en el marco de políticas para la reducción del riesgo por inundación, no ha sido suficientemente explorado en investigaciones metropolitanas, y específicamente, en investigaciones orientadas a la formulación de acciones, estrategias y modelos de adaptación y mitigación (Jáuregui et al., 2017).

Hay una carencia general en los estudios que investigan el riesgo hídrico de una orientación asociada a la percepción social de la inundación, más aún de estudios que analicen la percepción a escala regional, lo cual es posible a través del análisis de un medio de difusión masivo como el diario El Día, el periódico con mayor difusión de la región.

En este marco, el objetivo del presente artículo es avanzar en pos de la gestión colectiva del riesgo, generando aportes dentro 
de las políticas de identificación, en relación a la representación social del riesgo. Se enmarca en una línea de investigación en desarrollo que pretende analizar y explicar las características que asume la problemática de las inundaciones en la microrregión GLP, como amenaza territorial y ambiental, para llevar a cabo una primera aproximación hacia la construcción de una matriz de riesgo por inundación.

El presente trabajo consiste en identificar los procesos de vulnerabilidad ambiental por inundación en la microrregión mediante la detección de eventos y su localización - ya sean tormentas o sudestadas - publicados en artículos periodísticos entre los años 1911 y 2014, con la posterior sistematización y análisis.

En una primera parte se presenta el caso de estudio, profundizando en las características que asume el riesgo en la región y evidenciando la necesidad de hallar mecanismos capaces de involucrar a la población afectada en todas las etapas que comprende la gestión colectiva del riesgo, incluyendo la difusión. A continuación se presentan los datos obtenidos en base a la metodología en la microrregión y se discuten los resultados. A modo de reflexión final se analiza la importancia de implementar mecanismos que recojan la percepción social del riesgo.

La metodología desarrollada podría aplicarse a otros territorios y/o fenómenos que representen riesgos, que cuenten con periódicos locales, con el fin de reconstruir los antecedentes de la vulnerabilidad. Esto significa que, más allá del cambio de paradigma - hoy a diferencia de ayer, se reconoce que las actividades del hombre son el centro de las modificaciones de los procesos naturales y no al revés, como se lo consideraba antes de 1970 - al registrarse estos antecedentes, su análisis podría colaborar a encontrar otras explicaciones exentas de una mirada catastrófica, que hoy ya resulta anacrónica.

En este sentido Poggiese (2004, p. 232) advierte que

[...] no hay desastres naturales sino que, aun teniendo origen en fenómenos naturales, tecnológicos, antrópicos, etc., los desastres son, en esencia, una construcción social, el resultado de un proceso de ocupación y de producción que, como 'desarrollo', ha vinculado la vida humana con la naturaleza. En verdad se llega al desastre por un estado de exposición anterior al evento: el desastre no es otra cosa que la afirmación de las cuentas negativas del desarrollo adoptado.

La exposición a este tipo de eventos cada vez más frecuentes, requiere de la interdisciplina, la acción multisectorial y la participación social; la interrelación de actores, así como la interacción entre Estado y sociedad son sustanciales para enfocarse en la resolución, mitigación y difusión de los desastres y conformar la agenda de las políticas públicas antes de producida la catástrofe.

\section{El fenómeno de las inundaciones urbanas en el Gran La Plata}

El GLP se desarrolla en una extensión de 1162 km² y posee una población de 799.423 habitantes (Indec, 2010). Se ubica sobre el litoral del Río de la Plata en la zona sur de 
Figura 1 - Acercamiento al área de estudio

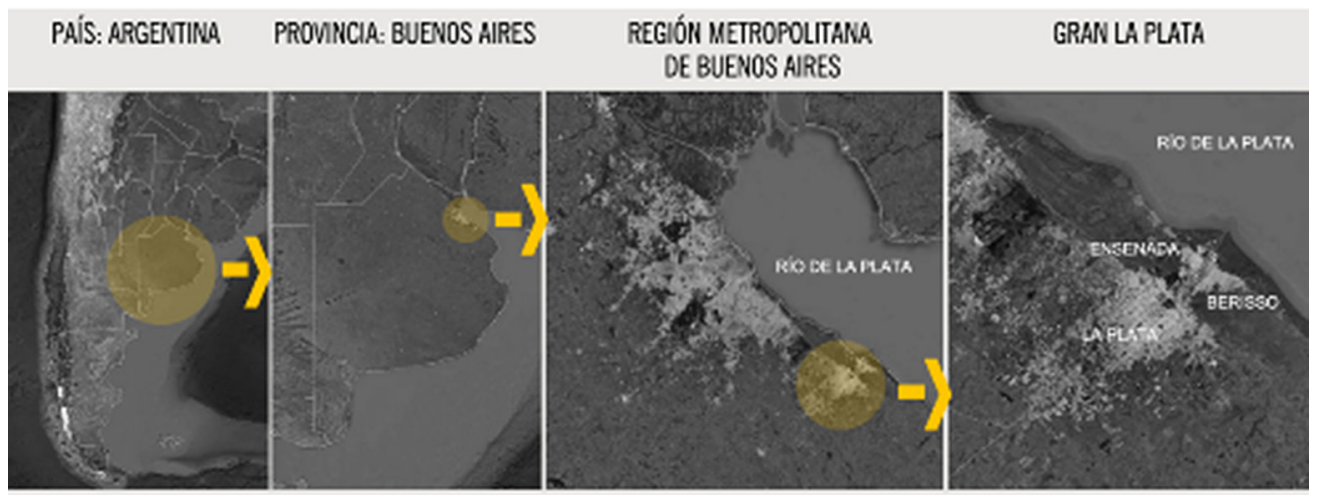

Fuente: elaboración propia en base a Google Earth.

la Región Metropolitana de Buenos Aires (Figura 1).

Los partidos de Berisso y Ensenada se desarrollan sobre el frente fluvial, como primeros asentamientos del conglomerado de origen industrial y portuario, mientras que la ciudad de La Plata fue fundada como ciudad planificada en 1882 por la necesidad de contar con una ciudad capital para la provincia de Buenos Aires, dos siglos más tarde que el primer poblado de La Ensenada de Barragán. La Plata se ubica en la terraza alta, distanciada de las anteriores por el Bañado Maldonado.

De esta manera, si bien los tres partidos son diferentes jurisdicciones (a partir del año 1957), conforman un ambiente asentado en un medio físico que los integra sobre el frente costero; asimismo los tres mantienen profunda vinculación por las interrelaciones entre los lugares de trabajo, vivienda y ocio, lo cual permite caracterizar al conglomerado como una microrregión. Este medio físico ocupa la pampa ondulada interior (en la terraza alta a +5 mtrs. sobre el nivel del mar), está configurado por cuencas y subcuencas, atravesado por arroyos (canalizados, entubados y/o a cielo abierto) que desaguan a través del bañado Maldonado en el Río de la Plata, configurando una planicie de inundación, con las implicancias que esto genera en términos de riesgo.

Dicha planicie es una restricción del medio natural para el asentamiento humano, en la franja litoral. Sus particularidades están dadas también por los suelos arcillosos, anegamientos permanentes, napa freática salinizada y cerca de la superficie (Galafassi, 1998). Además de ser un activo ambiental que regula el escurrimiento del agua.

Durante el proceso de configuración del sector, los arroyos fueron conformando barreras, drenajes, conductos, espacios de evacuación de efluentes, causando también inundaciones, pero no fueron tratados contemplando la lógica de la ocupación de cada una de las subcuencas durante el proceso de ocupación.

Como se ha comentado brevemente en la introducción, hay dos amenazas principales asociadas al riesgo de inundación en la microrregión: el fenómeno denominado como 
"Sudestada", que afecta centralmente a los municipios costeros de Berisso y Ensenada, que se desencadena cuando los vientos procedentes del cuadrante Sur-Sudeste impiden el escurrimiento de las aguas del Rio de La Plata, produciendo la elevación del nivel del río y la interrupción del escurrimiento regular de los afluentes; y por otro lado las tormentas periódicas que afectan sobre todo a las cuencas de los arroyos tributarios del Río de La Plata, siendo las áreas más afectadas las planicies de inundación que han sido progresivamente ocupadas por el proceso de urbanización, en particular en la ciudad de La Plata (Figura 2).

El territorio en estudio por lo tanto se configura como un ambiente vulnerable; siendo parte de un asentamiento litoral que además aloja actividades incompatibles que compiten por el uso de los recursos suelo y agua; un territorio de interfase de carácter frágil a partir de la ocupación de las planicies de inundación, con problemas de inundaciones cíclicas, impacto negativo de las industrias y enterramiento de residuos sólidos (López y Etulain, 2017).

Reflejo de la vulnerabilidad del GLP, son algunas de las inundaciones que se produjeron en la región. Entre ellas, las que ocasionaron mayores impactos en los últimos años fueron las ocurridas en 2002, 2006, 2008 y 2013. Con respecto a esta última un informe de la Facultad de Ingeniería de la Universidad Nacional de La Plata manifiesta que

[...] su magnitud se ubicó por encima del máximo histórico registrado. A consecuencia de ello, se excedieron las capacidades de los arroyos en cuyas cuencas se asienta fundamentalmente la ciudad de La Plata. Las aguas, al extenderse hacia sus propias planicies

Figura 2 - Registros por partidos. La Plata, Berisso y Ensenada

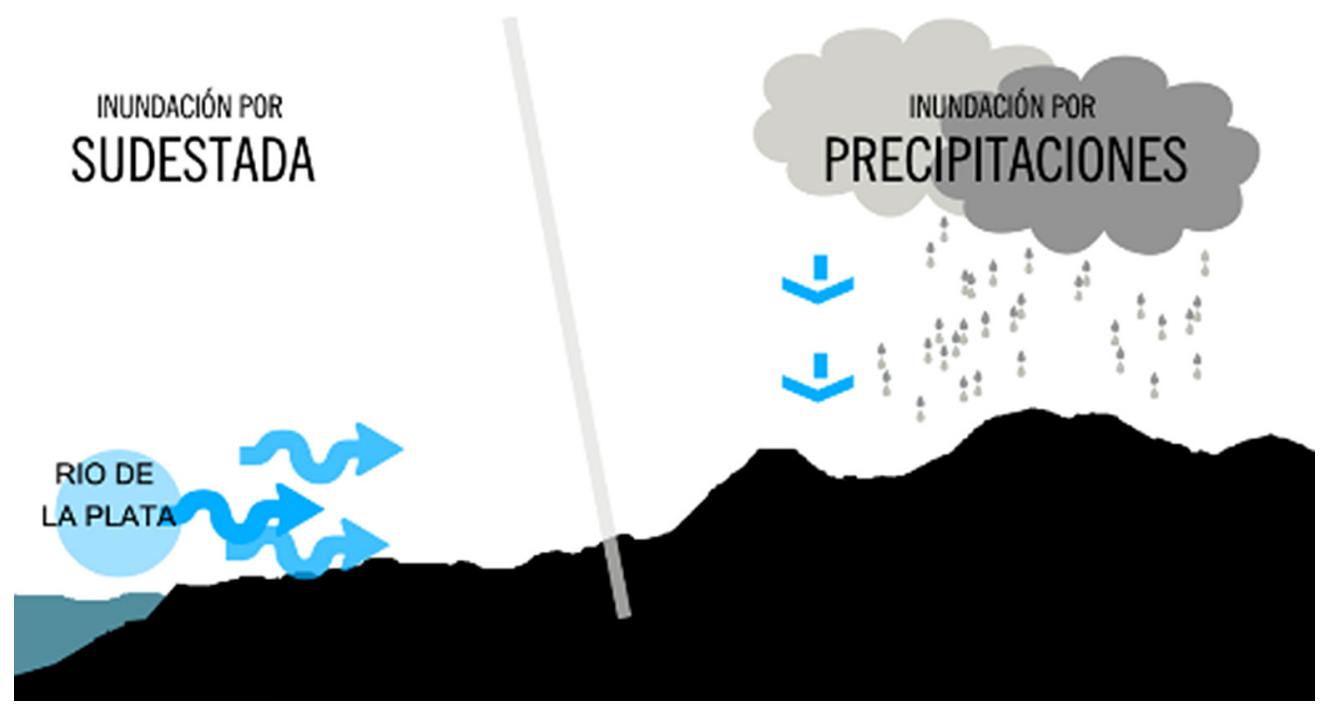

Fuente: elaboración propia. 
de inundación y reocupar las huellas de sus antiguos cauces, produjeron el anegamiento de amplias zonas de la ciudad. (Facultad de Ingeniería, 2013)

La combinación de los fenómenos de sudestada y precipitaciones extraordinarias con una urbanización de llanura - atravesada por lo menos por diez arroyos - asentada en sus bordes y planicies de inundación convierte a la sociedad en vulnerable. Reconocer las áreas con mayores porcentajes de exposición a inundaciones permite - además de generar un diagnóstico - definir líneas de acción, de gestión y ejecución de estrategias para mitigar tales impactos.

Este trabajo plantea la carencia en el abordaje del estudio de la percepción social del riesgo, resulta insuficiente el análisis del cambio climático y la idea de que la naturaleza del evento es la que conduce al desastre, concepción que está quedando superada por el cambio de percepción en la sociedad.

En este sentido el aporte innovador de este trabajo consiste en una metodología que articula el mapeo del riesgo a través de la percepción social del mismo, relevada a partir de artículos periodísticos, lo cual significa un enfoque innovador al análisis del riesgo en tanto reúne un medio masivo de difusión local-regional, la participación ciudadana y el análisis de los eventos.

\section{Metodología}

La construcción de la metodología se basó en el análisis documental, a partir del registro de artículos periodísticos publicados en el período antes mencionado (tomando como fuente el principal y más antiguo medio gráfico de la región -"El Día"), su sistematización en tablas y gráficos, y su posterior espacialización y elaboración de cartografía.

La metodología se estructuró fundamentalmente en tres etapas articuladas. La primera consistió en la búsqueda de artículos periodísticos relativos a eventos climáticos de precipitaciones (tormentas y sudestadas), con efectos de anegamientos o inundaciones en las diferentes localidades de la microrregión, para los años transcurridos entre 1999 y 2014 inclusive. Se contaba además con un registro para los años 1911 a 1998, ${ }^{3}$ procedente de la misma fuente, que se procesó como antecedente (con las limitaciones propias de no haber contado con un motor de búsqueda digital para ese período).

En la segunda etapa, se procedió a la sistematización de estos artículos a partir de la construcción de una tabla síntesis. Para esto fue necesaria la construcción de un protocolo de carga de datos que permitiera posteriormente georreferenciar y espacializar los mismos en el sistema de información geográfica (en adelante: SIG) de software libre GvSig - entendido como una "base de datos georreferenciada, diseñada para visualizar, editar, gestionar y analizar información geográfica con el fin de contribuir a resolver problemas complejos de planificación y gestión del territorio" (Dirección Provincial de Ordenamiento Urbano y Territorial, 2011, p. 15), que permitiera el trabajo interrelacionado de las variables.

Se construyó una tabla en la que se sistematizaron los registros de artículos periodísticos del período 1999-2014 (Figura 3) 
del Diario El Día de la ciudad de La Plata; en la que se recogieron los siguientes datos:

(1) código de la noticia (diseñado para poder identificar individualmente cada una de las noticias);

(2) título del artículo periodístico (otorgado por el propio medio periodístico);

(3) link del artículo (que conduce a la página web del artículo periodístico);

(4) tipo de evento (indicando si se trató de un evento producido por "tormenta" o por "sudestada");

(5) fecha del evento (señalando día, fecha y mes en que se produjo el evento),
(6) pluviometría (cantidad de lluvia, medida en milímetros);

(7) altura máxima que alcanzó el agua en calle (siempre que está información estuviera disponible en el artículo periodístico);

(8) partido, localidad y barrio (para reconocer el área en la que se produjeron los eventos);

(9) zonas anegadas (a partir del registro, y posterior mapeo, de encrucijadas de calles); y efectos (pérdidas materiales, evacuados, fallecimientos, entre otros).

Como parte de esta segunda etapa, se realizó un análisis sobre los datos obtenidos, con el fin de determinar los años críticos sobre

Figura 3 - Registro de artículos periodísticos. Campos Utilizados

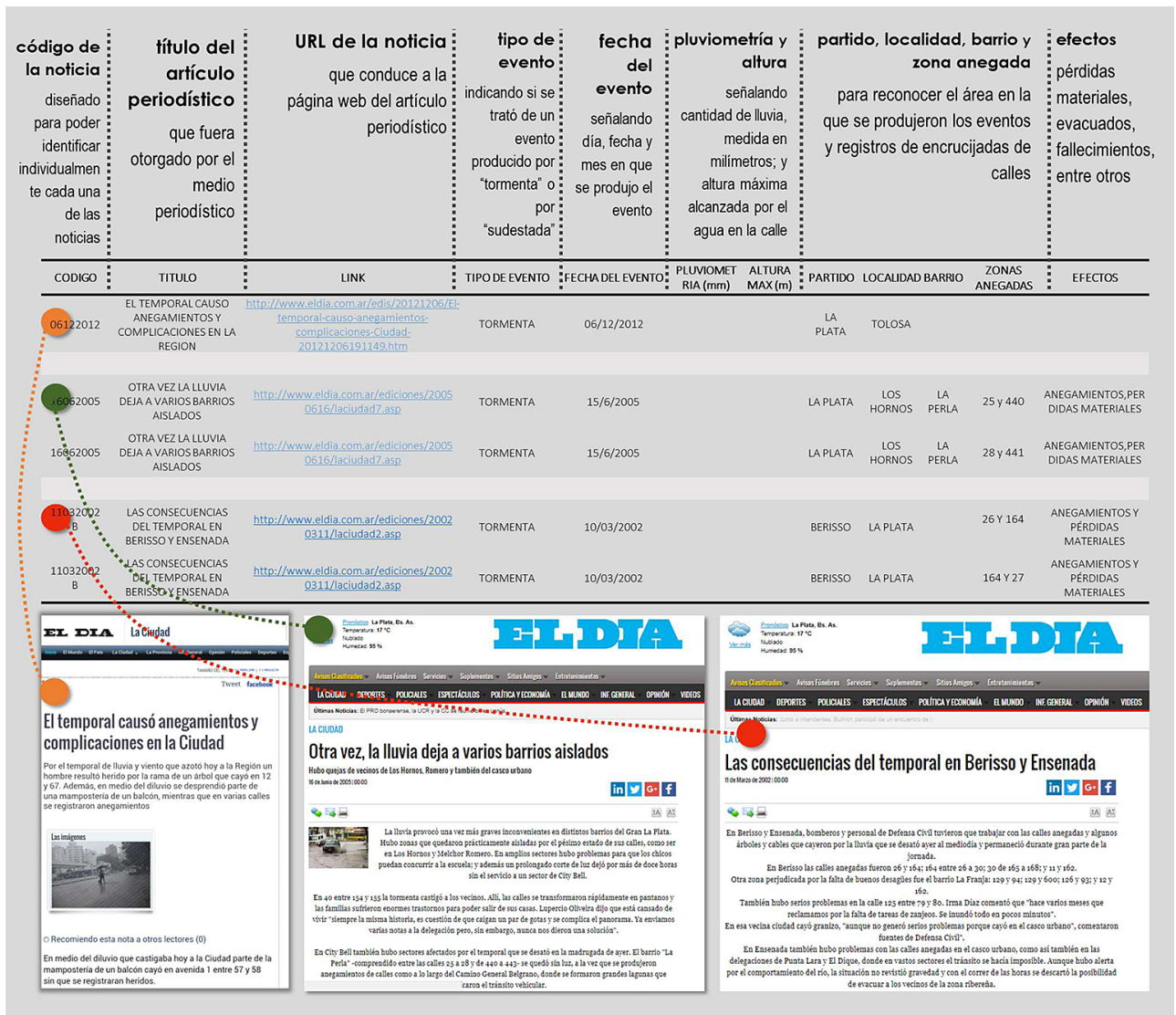

Fuente: elaboración propia. Fuente de las imágenes: https://www.eldia.com/ 
los que posteriormente se realizarían estudios pormenorizados. Así, quedaron definidos como tales los años 2002, 2006, 2008 y 2013, en virtud de registrarse en estos años los eventos de precipitación de mayor magnitud del período.

En la tercera etapa de trabajo se procedió a la georreferenciación y estudio de los datos obtenidos para los años mencionados, definiendo las áreas de mayor vulnerabilidad, y reconociendo que serán más vulnerables aquellas zonas en las que se reiteren los anegamientos en el período abarcado, o que, pese a que no se registre una reiteración de eventos, ante una situación extraordinaria el evento tenga efectos de mayor magnitud.

De esta forma, tomando como base la información sistematizada en la tabla, específicamente el campo de "zona anegada" en el que se indicaban las encrucijadas de las calles afectadas por los diferentes eventos, se realizó la espacialización mediante SIG, obteniendo mapas de puntos inundados.

Este insumo inicial, sumado a la información restante con la que se contaba en la tabla, permitió realizar una serie de análisis en base a diferentes criterios: tipo de evento (precipitación o sudestada), año de los eventos, pluviometría, repetición de zonas anegadas, entre otros.

Asimismo, la digitalización a partir de un SIG, permite articular esta información con otras variables fundamentales para el análisis, incorporando no sólo datos meteorológicos, sino también "Tipo de evento" (sudestada o tormenta), "Altura de agua en calle", "Efectos del evento". Esta metodología, en construcción, significa un punto de partida para considerar y valorar el rol de la población en la construcción social del riesgo a través de la exposición en medios gráficos, televisivos, radiales y redes sociales. Desde allí, la metodología permite explorar la formulación de diversos productos, de carácter dinámico e interactivo.

\section{Resultados y discusion}

Los datos presentados a continuación sistematizan los resultados obtenidos en base al registro y mapeo de inundaciones entre los años 1999 a 2014 en relación al tipo de evento y a los partidos y localidades afectadas, así como un análisis comparativo de puntos inundados en los años críticos mencionados.

\section{Tipo de evento}

En principio se estudió el tipo de evento en los diferentes registros, diferenciando si se trataba de inundaciones producidas por sudestada 0 por precipitaciones (Figura 4). De esta forma, pudo observarse que los mayores registros de eventos estaban asociados a situaciones de precipitaciones (3588 eventos) y no así a "sudestadas" (271 eventos), siendo que se trata de una zona con características costeras y podría interpretarse que este fenómeno meteorológico generaría las mayores problemáticas. Puede interpretarse también una relación entre los mayores registros y la localización de la sede del diario, asimismo la accesibilidad del diario para registrar los eventos, fotografiarlos y entrevistar a los usuarios perjudicados. 
Figura 4 - Tipo de evento registrado

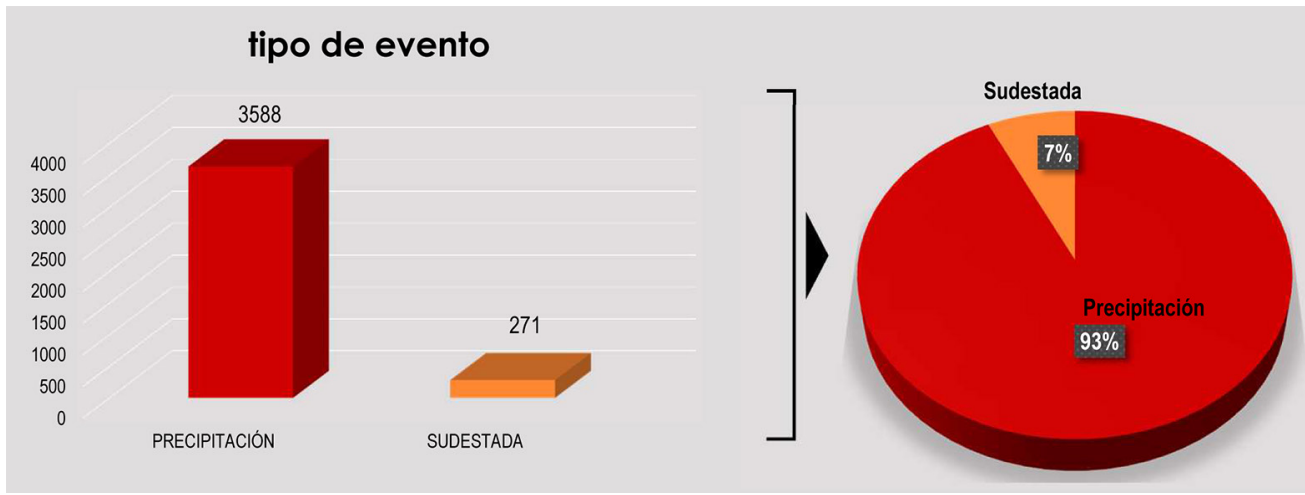

Fuente: elaboración propia.

Sobre el total de los registros hallados (3859 eventos), el 93\% corresponde a anegamientos o zonas inundadas por precipitación y el $7 \%$ a sudestada. Este último fenómeno es conocido por los lugareños de los ámbitos costeros y los frentes ribereños, quienes suelen tomar prevenciones ex ante, por lo que el evento es más previsible. Mientras que las inundaciones por precipitación son más sorpresivas y en ocasiones en zonas infrecuentes, conduciendo a la necesidad de darlo a conocimiento al periódico.

En este contexto, también se puede asociar que las noticias están vinculadas a la percepción social del riesgo; se mencionó previamente que la población que se ve afectada por las sudestadas está acostumbrada, representa lo que Herzer y Merlinsky (2012, p.15) denominan "riesgo físico", que diferencia del "riesgo socialmente reconocido" siendo este último el que se vincula a la acción política.

\section{Registro por partido}

Con respecto a las zonas anegadas, se realizaron dos tipos de análisis: en principio un análisis general, en relación a cuál de los partidos de la región GLP se vio más afectado por los diferentes eventos; y en una segunda instancia un análisis particularizado, en relación a las localidades que registraron mayor cantidad de puntos.

El análisis general permitió reconocer que de los tres partidos que conforman la región $\mathrm{GLP}$, los mayores registros se observan en el partido de La Plata (3198), seguido por el partido de Berisso (442 registros) y finalmente por el partido de Ensenada (219 registros). Considerando la totalidad de los registros, La Plata representa el $83 \%$, Berisso el $11 \%$ y Ensenada sólo un 6\% (Figura 5).

Tal cual como sucede con el análisis del primer punto, se puede inferir que la cantidad de afectados es mayor en el partido de La Plata 
Figura 5 - Registros por partidos. La Plata, Berisso y Ensenada

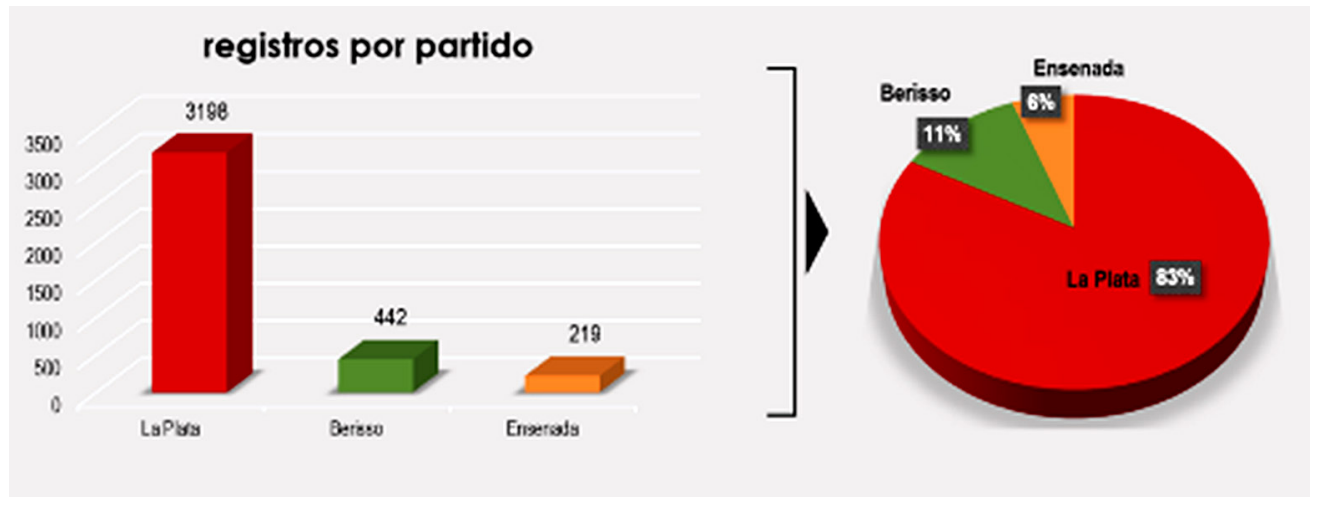

Fuente: elaboración propia.

considerando que es el partido con mayor población y es proporcionalmente coincidente. Del mismo modo, los usuarios del diario que tienen más acceso están en el partido de La Plata. Los partidos de Berisso cuentan con medios locales que si se analizaran con la misma metodología podrían ampliar el análisis.

\section{Registro por localidad}

El análisis particularizado, permitió reconocer, según el registro de puntos afectados por año y localidad (Figuras 6 y 7), que el casco de la ciudad de La Plata contaba con la mayor cantidad de puntos afectados en un rango de 151 a 650 (totalizando 644 puntos registrados), seguido por las localidades de la periferia inmediata (Villa Elvira, Los Hornos, Ringuelet, Tolosa y San Carlos) todas en un rango entre 41 y 150 puntos. En un tercer grupo se sitúan las localidades de la periferia no lindante al casco (Villa Castells, Melchor Romero, Arana, Gonnet, City Bell, Altos de San Lorenzo, Villa
Elisa, Ensenada, Gorina, Lisandro Olmos) y los partidos de Berisso y Ensenada en un rango de entre 0 y 40 puntos registrados. En este último grupo se encuentran otras localidades, con la menor cantidad de puntos afectados (El Dique, Abasto, Villa Argüello, Hernández, Arturo Seguí y el Barrio Aeropuerto).

Lo que se evidencia es que la accesibilidad al medio local es decreciente tomando el casco histórico como centro hacia los bordes periféricos. Esto se puede deber a diferentes circunstancias, una de ellas, y según consta en algunas noticias relevadas, son los cortes de luz que afectan áreas periféricas y que se mantienen por plazos prolongados, lo que dificulta la posibilidad de contar al medio el evento y sus consecuencias; otra situación que surge del cruzamiento de datos de población, NBI (Indice de Necesidades Básicas Insatisfechas) e infraestructuras indica que las poblaciones más vulnerables que además se asientan en lugares con mayor riesgo de inundación, en el momento que se produce el evento y ex post tienen 
que atender las cuestiones esenciales de supervivencia, evacuar, sacar el agua, salvar los recursos materiales, antes que preocuparse por advertir al diario sobre la ocurrencia del evento. Siguiendo a Herzer y Di Virgilio (1996, p. 66) "los sectores de menores recursos se asientan, generalmente, en tierras de escaso valor inmobiliario, con importantes déficits de infraestructuras y servicios, la localización de la población pobre en las áreas periféricas de las ciudades expresa procesos de segregación y desigualdad urbana".

Figura 6 - Registro de eventos (por cantidad de artículos) identificados por delegación. Partidos de Berisso, Ensenada y La Plata

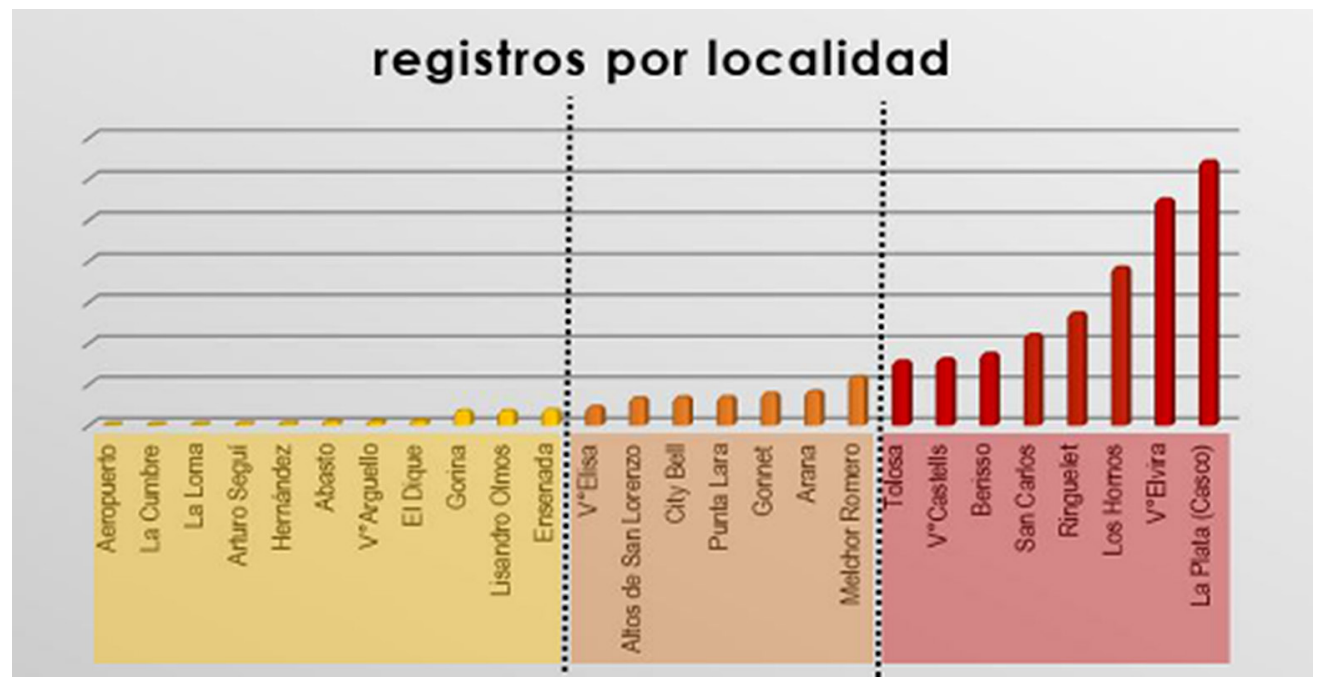

Fuente: elaboración propia. 
Figura 7 - Registro de eventos identificados por delegación. Partidos de Berisso, Ensenada y La Plata

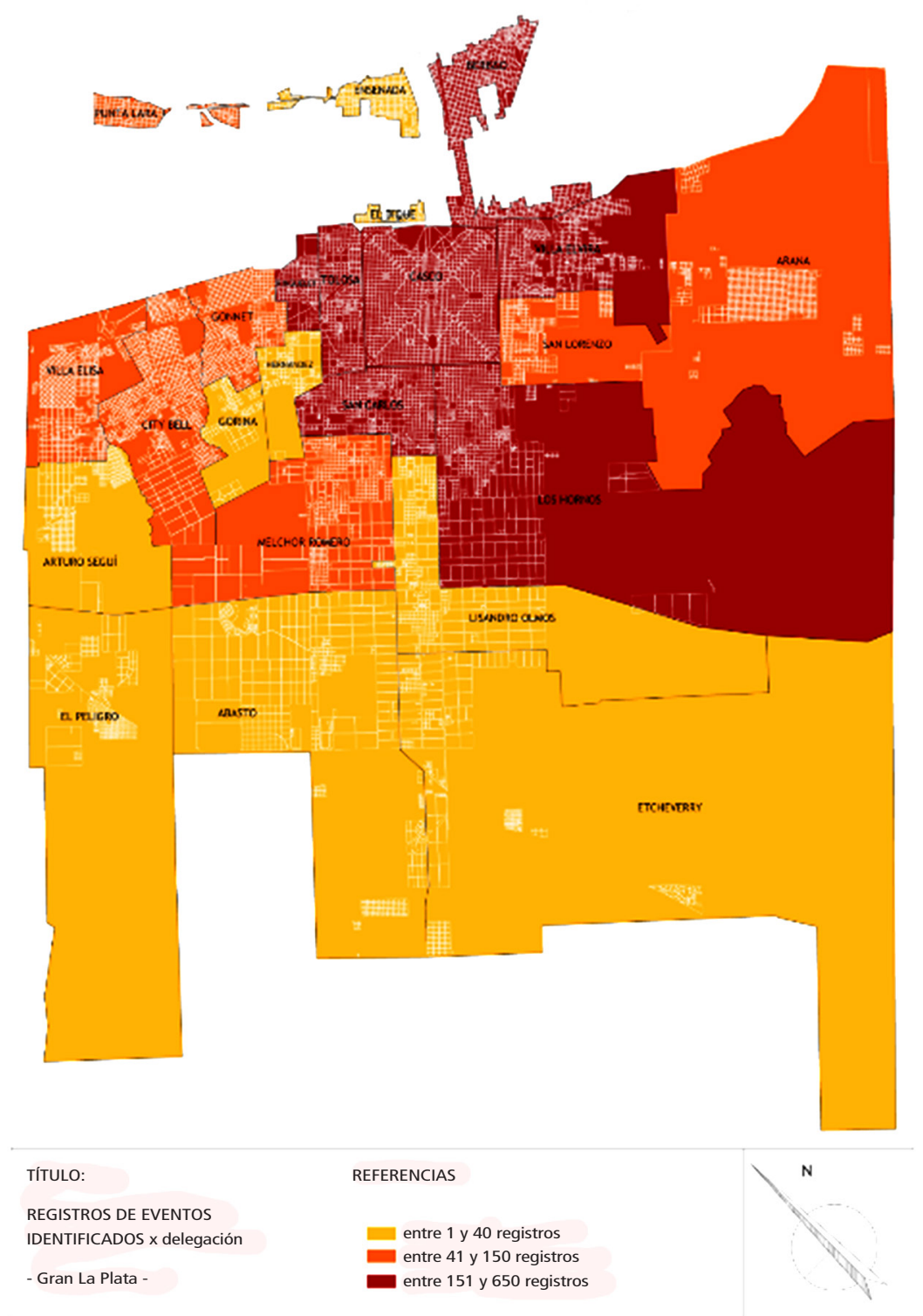

Fuente: elaboración propia. 


\section{Años críticos}

En base a investigaciones realizadas previamente, pudo reconocerse que los períodos mapeados coincidieron con las inundaciones más cuantiosas que se produjeron en la zona. Así, se detectó que las localidades que presentaban mayor cantidad de registros para la totalidad del período analizado (Casco, Villa Elvira, Los Hornos, Ringuelet y San (arlos), coincidían parcialmente con las áreas definidas como más vulnerables. Con la información y espacialización de los eventos (en forma puntual) reconocidos para estos años se realizó una superposición y entrecruzamiento de información, con el objeto de identificar patrones y situaciones particulares (Figura 8). A continuación se exponen los mapas construidos para el año 2002, 2006 y 2008 donde pueden observarse patrones de inundación bien diferenciados.
En el análisis del año 2002 se observa que los puntos de registros se concentran en áreas específicas, conformando zonas anegadas en la localidad de Punta Lara en el partido de Ensenada, en el partido de Berisso y fuera del casco sobre los ejes de la red vial de estructura metropolitana y local hacia el sudeste, hacia el sudoeste y noroeste (sobre Avenida 7, Caminos Centenario y Belgrano). Sin embargo, los puntos dentro del casco son dispersos.

En el análisis del año 2006, sin embargo las concentraciones de puntos registrados no siguen el patrón del año 2002, hay dispersión de puntos, aunque aparecen como localidades afectadas nuevamente Villa Elvira y sobre los ejes de red vial metropolitana y local pero con mayor énfasis hacia la zona norte en localidades de City Bell y Villa Elisa.

En el análisis del año 2008, los registros encontrados se localizan desde el centro del

Figura 8 - Espacialización de años críticos 2002/2006/2008
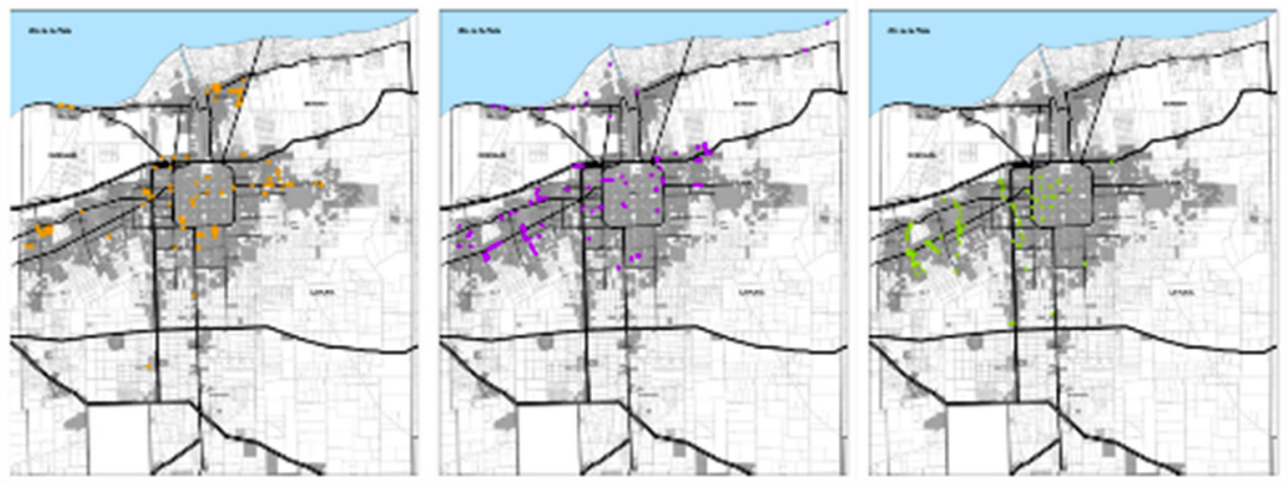

Fuente: elaboración propia. 
casco hacia el norte, fundamentalmente en las localidades Gonnet y City Bell, debido que la cantidad de agua caída no es homogénea como tampoco su permanencia, siendo mayor en estas zonas generando importantes anegamientos; no habiendo registros sobre el borde costero en los partidos de Ensenada y Berisso.

Sintetizando los años críticos se puede decir que toda la región está sometida a la ocurrencia de precipitaciones con consecuencias de anegamientos e inundaciones. Sin embargo revisando el análisis de los puntos anteriores se puede advertir que la vulnerabilidad no depende únicamente del evento, sino de la ocupación y usos del suelo, el grado de NBI, la densidad poblacional, la accesibilidad, infraestructuras y equipamientos urbanos, por una cuestión de espacio no se describen aquí los elementos que conforman el diagnóstico de base para este tipo de análisis. Como menciona Herzer (2011, p. 53)

[...] una visión amplia sobre el medio ambiente urbano debería partir del reconocimiento de la fragmentación social de la ciudad, identificando tres puntos fundamentales: 1) las relaciones entre la sociedad y su medio; 2) las relaciones entre diversos actores sociales; 3) las articulaciones entre distintos niveles jurisdiccionales y sectoriales.

\section{Conclusiones}

Los resultados presentados en cuanto a tipo de evento, registro por partido y localidad, y patrones de inundación en distintos años críticos, avanzan en la gestión colectiva del riesgo en la microrregión, desde la identificación de distintos aspectos del riesgo de inundación que relevan la percepción social que se plasma en el periódico relevado.

Respecto del tipo de evento, el hecho de que más del $90 \%$ de los registros de inundación sean por precipitación y que más de un $80 \%$ se registre en la ciudad de La Plata, contradice cierta idea presente en el imaginario colectivo, de que las inundaciones se asocian a la situación costera de la microrregión. Dejando en evidencia el desconocimiento de la población acerca del medio físico donde la ciudad se asienta, que a pesar de desarrollarse en un sector alto respecto al nivel del río, es un área surcada por múltiples planicies de inundación, que convierten en vulnerable a la población allí asentada. La conciencia social acerca de los arroyos que discurren entubados por áreas las urbanas consolidadas surgió a partir de la inundación del año 2013, a partir de que las aguas reocupen sus antiguos cauces, sin embargo es un tema que hay que continuar reforzando para crear prevención y que no sea únicamente, y tardíamente, tratado en ocasión de una inundación de gran magnitud.

En base al registro por localidad es posible establecer tres rangos de valores que van de centro a periferia de la ciudad de La Plata, lo que obliga a asociar los puntos inundados a otros indicadores tales como NBI, acceso a las infraestructuras de comunicación, necesidades distintas ante una emergencia. Cabe mencionar que se han registrado omisiones mediáticas sobre las áreas inundadas que concentran asentamientos informales (villas). Esto se expresa en particular en las localidades de Altos de San Lorenzo, Los Hornos, Lisandro 
Olmos, y en el eje portuario-industrial (zona interjurisdiccional) (Jáuregui et al., 2015).

En este sentido es necesario realizar un análisis integral del riesgo superando la visión dominada por la peligrosidad y la exposición, dimensiones que según señala Natenzon (2003) han primado en Argentina respecto de la búsqueda de soluciones para las inundaciones, frente al análisis de la incertidumbre y de la vulnerabilidad, esta última asociada a la situación socioeconómica de la población.

El análisis de los años críticos permite encontrar puntos donde las inundaciones se reiteran, así como patrones de inundación diferenciados en cada año, lo que impulsa a analizar la localización y la dinámica de la amenaza, en este caso de las precipitaciones, un tema dificultoso en la región a partir de la falta de una red de estaciones de medición que cubra una mayor extensión territorial. Asimismo, recientemente las estaciones han sido equipadas para poder medir pluviometrías como la registrada en 2013 , donde una sola de ellas pudo medir la lluvia en esa ocasión. Esto le da aún más relevancia al estudio según artículos de periódicos de las zonas inundadas, que resultan un archivo amplio de registros pluviométricos, inundaciones y daños producidos a lo largo de más de 100 años.

Con este registro y acervo analítico en torno a las inundaciones de la microrregión, se avanza a modo de reflexión en la interacción que existe entre el evento como dato objetivo, sea inundación o sudestada, la difusión en modo de alertas y de consecuencias y la percepción que la población tiene sobre el fenómeno ex ante y ex post en la construcción social del riesgo, vinculaciones fundamentales a la hora de construir conciencia social acerca de los fenómenos que afectan a la región y contribuir a la gestión integral del riesgo.

Sin dudas, de cara a la mejora del método de registro y mapeo de inundaciones a través de medios de comunicación, es necesario contar con otras fuentes de información, además del principal periódico de la microrregión. En principio es necesario incluir a periódicos locales de los partidos de Berisso y Ensenada, para relevar el impacto de las inundaciones por precipitaciones en estos partidos, así como para contar con otros registros de las inundaciones por sudestada.

Por ejemplo, en el caso de la ciudad de La Plata se observaron más puntos que en los otros municipios, pero este dato no está necesariamente en relación con lo ocurrido sino en relación a la relevancia que un medio da a una ciudad u otra, y en relación a como la población local-regional interactúa con el medio. Este es otro punto relevante para este tipo de trabajos porque esta metodología repetida en el resto de los medios locales daría un panorama más extendido y representativo del riesgo al que la sociedad se expone.

Este trabajo ha orientado la búsqueda de una metodología que aporte a la vinculación de la esfera académica con el medio social a través de las nuevas formas de información y comunicación. Esos medios pueden ser las redes sociales, la universidad, los periódicos u otros nuevos, que estén en línea con lo dicho en este artículo. Existe una demanda de la sociedad por conocer en profundidad las posibilidades de ocurrencias del fenómeno como parte de la agenda que el Estado debería garantizar a través de la difusión en medios masivos de comunicación y por sistemas de alertas tempranas según el servicio 
meteorológico nacional; en coincidencia con Ascher (2004, p. 68) "es típico de la sociedad moderna querer controlar el futuro, y para ello intenta conocer y medir las probabilidades de que se produzca tal o cual acontecimiento para tomar después decisiones al respecto".

En ese sentido en las sociedades en vías de desarrollo donde hay muchas limitaciones y carencias por suplir, parece imprescindible el rol social de los medios de comunicación y redes sociales en la información, difusión y comunicación; como advierte Castells (1997، p. 399)

[...] la integración potencial de texto, imágenes y sonido en el mismo sistema, interactuando desde puntos múltiples, en un tiempo elegido (real o demorado) a lo largo de una red global, con un acceso abierto y asequible, cambia de forma fundamental el carácter de la comunicación y ésta determina decisivamente la cultura.

Lo que se evidenció en la microrregión, el día de la última gran inundación de 2 y 3 de abril de 2013 y de las anteriores, respecto del evento, la vulnerabilidad y el riesgo, es una falta de conocimiento, además de la falla, o inexistencia, en el pre-aviso de la ocurrencia de un evento de magnitud significativa. Un importante desconocimiento de la configuración física del territorio: esto implica que la población urbana, principalmente de la ciudad de La Plata expuesta a la inundación no estaba en conocimiento del sistema de drenajes naturales, entubados, que surcan la ciudad y se escurren por debajo de los inmuebles.

Existe una carencia de información acerca de este tipo de fenómenos meteorológicos. La población en general y la urbana céntrica en particular no pensaba que esta circunstancia iba a empeorar con el transcurso de las horas, por el contrario las diversas notas y charlas con la gente dan cuenta que en su mayoría tenían certezas que el agua iba a bajar, sin embargo aun con menor cantidad de agua caída el agua siguió aumentando porque el sistema de drenajes no evacúa sino que desborda.

Además, los sistemas de prevención de las áreas reconocidas como vulnerables no alcanzaron: en la microrregión y en la ciudad de La Plata hay sectores con mayor vulnerabilidad frente al fenómeno, esa población se encuentra más alertada y toma medidas de prevención (sistemas de bombeos, compuertas, superficies más elevadas), lo que en esta última ocasión no sirvió para contener el agua, ya que la altura superó lo conocido por la experiencia previa. Desinformación previa, durante y posterior al evento: qué hacer, a dónde ir, a quién recurrir, cómo autoevacuarse fueron cuestionamientos generales por la falta de información respecto a la contingencia. Este punto es fundamental en el sentido que este tipo de fenómenos es, y será, cada vez más frecuente, y es sustancial que la población conozca vías de escape, formas de evacuación, pautas de mitigación y/o formas de protección.

Los resultados expuestos y los debates que se desprenden de ellos demuestran que la percepción del riesgo es como denomina Ascher (2004, p. 68) "una noción típica de la modernidad", al mismo tiempo que se conocen las vulnerabilidades y se exige mayor información y acción, se desatiende el crecimiento de las ciudades y el ordenamiento en línea con el futuro poco favorable para las ciudades asentadas en planicies de inundación y sobre frentes costeros. 
Ante este escenario y frente a la ausencia del Estado en la prevención del riesgo, la única posibilidad de la sociedad es la sobreponerse y esperar: generar resiliencia. Sin embargo en pos de la gestión integral del riesgo, debiera ser el estado a través de una agenda de políticas públicas, conjuntamente con la sociedad, quienes construyan un escenario futuro deseable en relación al modelo de desarrollo sostenible que plantea Naciones Unidas, teniendo en cuenta además, que las inundaciones son el riesgo de desastres más importante en Argentina (Secretaría de Ambiente y Desarrollo Sustentable, 2015).

[I] https://orcid.org/0000-0003-1571-2396

Consejo Nacional de Investigaciones Científicas y Técnicas, Universidad Nacional de La Plata, Facultad de Arquitectura y Urbanismo, Centro de Investigaciones Urbanas y Territoriales. La Plata, Argentina.

rotgerdaniela@hotmail.com

[II] https://orcid.org/0000-0003-0057-391X

Consejo Nacional de Investigaciones Científicas y Técnicas, Universidad Nacional de La Plata, Facultad de Arquitectura y Urbanismo, Centro de Investigaciones Urbanas y Territoriales. La Plata, Argentina.

mma_arq@yahoo.com.ar

[III] https://orcid.org/0000-0002-4339-5044

Universidad Nacional de La Plata, Facultad de Arquitectura y Urbanismo, Centro de Investigaciones Urbanas y Territoriales. La Plata, Argentina.

jaureguiestefania@gmail.com

\section{Notas}

(1) Las Inundaciones en La Plata, Berisso y Ensenada: Análisis de Riesgos y Estrategias de Intervención. Hacia la construcción de un Observatorio Ambiental. Proyecto de Investigación Orientado PIO UNLP-Conicet 2014-2017. Directora del proyecto: Dra. Alicia Ronco. Codirectora: Arq. Isabel López.

(2) El equipo que dentro del proyecto desarrolló el registro histórico de las inundaciones ha estado formado por: Estefanía Jáuregui, María Aversa, Daniela Rotger, Kuanip Sanz Ressel, Natalia Amor y Augusto Ávalos.

(3) Realizado por la arquitecta Isabel López. 


\section{Referencias}

ASCHER, F. (2004). Los nuevos principios del urbanismo. Madrid, Alianza editorial.

CARDONA, O. D. (1993). “Evaluación de la amenaza, la vulnerabilidad y el riesgo”. En: MASKREY, A. (ed.). Los desastres no son naturales. Red de Estudios Sociales en Prevención de Desastres en América Latina LA RED-USAID.

(2001). La necesidad de repensar de manera holística los conceptos de vulnerabilidad y riesgo. Una crítica y una revisión necesaria para la gestión. En: INTERNATIONAL WORK-CONFERENCE ON VULNERABILITY IN DISASTER THEORY AND PRACTICE. Disaster Studies of Wageningen University and Research Centre. Wageningen, Holanda.

CASTELLS, M. (1997). La era de la información: economía, sociedad y cultura. Madrid, Alianza editorial.

DIARIO EL DÍA. Disponible en: https://www.eldia.com/. Acceso en: 3 junio 2018.

DIRECCIÓN PROVINCIAL DE ORDENAMIENTO URBANO Y TERRITORIAL DE BUENOS AIRES, ARGENTINA (2011). Sistemas de Información geográfica para el ordenamiento territorial. Disponible en: http:// www.mosp.gba.gov.ar/sitios/urbanoter/sig/Manual_SIG_UT.pdf. Acceso en: 15 enero 2018.

FACULTAD DE INGENIERÍA (2013). Estudio sobre la inundación ocurrida los días 2 y 3 de abril de 2013 en las ciudades de La Plata, Berisso y Ensenada. Disponible en: http://sedici.unlp.edu.ar/ handle/10915/27334. Acceso en: 15 enero 2018.

GALAFASSI G. (1998). Situación ambiental del Gran La Plata. Argentina. Definición de áreas aptas para urbanización. Revista Interamericana de Planificación - CIAP. Cuenca, v. XXX, n. 119 y 120, pp. 243-265.

HERZER, H. (2011). Construcción del riesgo, desastre y gestión ambiental urbana: perspectivas en debate. Revista Virtual Redesma, v. 5, n. 2, pp. 52-60.

HERZER, H. y DI VIRGILIO, M. M. (1996). Buenos Aires: pobreza e inundación. EURE, v. XXII, n. 67, pp. 65-80.

HERZER, H. y MERLINSKY, G. (2012). Ciudad, ambiente y construcción social del riesgo. Quid 16, n. 1, año 1, pp. 14-17.

INDEC - INSTITUTO NACIONAL DE ESTADÍSTICA Y CENSOS (2010). Censo nacional de Población y Vivienda. Disponible en: https://www.indec.gov.ar/nivel4_default.asp?id_tema_1=2\&id_ tema_2=41\&id_tema_3=135. Acceso en: 10 diciembre 2017.

JÁUREGUI, E.: AMOR, N.: SANZ RESSEL, K.; ROTGER, D.; AVERSA, M. e ÁVALOS, A. (2015). Ciudades Vulnerables: cartografias del riesgo hídrico en el Gran La Plata. En: XIX CONGRESO ARQUISUR. Actas. La Plata, Facultad de Arquitectura y Urbanismo. La Plata, pp. 1-8.

JÁUREGUI, E., AVERSA, M., y SALAS GIORGIO, R. (2017). Estrategias para la mitigación del riesgo por inundación. Caso Cuenca del Arroyo Maldonado, La Plata (Buenos Aires, Argentina). Urbano, n. 34, pp. 34-47.

LAVELL, A. (1996). “Degradación ambiental, riesgo y desastre urbano. Problemas y conceptos: hacia la definición de una agenda de investigación”. En: FERNÁNDEZ M. A. (comp.). Ciudades en riesgo. Degradación ambiental, riesgos urbanos y desastres. Red de Estudios Sociales en Prevención de Desastres en América Latina LA RED-USAID.

(2001). Sobre la gestión del riesgo: apuntes hacia una definición. Biblioteca Virtual en Salud de Desastres-OPS. Disponible en: http://www.undp.org. Acceso en: 10 diciembre 2017. 
LÓPEZ, I.; ETULAIN, J. C. et al. (2017). “Inundaciones urbanas: mapas de riesgo y lineamientos de ordenamiento urbano territorial”. En: RONCO, A. E. y LÓPEZ, I. (dir.). Las inundaciones en La Plata, Berisso y Ensenada: análisis de riesgo, estrategias de intervención. Hacia la construcción de un observatorio ambiental. Disponible en: http://sedici.unlp.edu.ar/handle/10915/59633. Acceso en: 2 diciembre 2017.

MASKREY, A. (1997). “Comunidad y desastres en América Latina: estrategias de intervención”. En: LAVELL A. (ed.). Viviendo en riesgo comunidades vulnerables y prevención de desastres en America Latina. Red de Estudios Sociales en Prevención de Desastres en América Latina LA RED-USAID.

NATENZON, C. (2003). Inundaciones catastróficas, vulnerabilidad social y adaptaciones en un caso argentino actual. Cambio climático, elevación del nivel medio del mar y sus implicancias. En: Climate Change Impacts and Integrated Assessment EMF (Energy Modeling Forum) Workshop IX. Stanford University. Snowmass, Colorado, pp. 1-16.

PÉREZ, R. (2013). Redes y centros urbanos bajo riesgo hídrico: prevención y mitigación de desastres naturales en planicies de inundación en la Argentina. Buenos Aires, Eudeba.

POGGIESE, H. (2004). Parte V “del ángulo de la gestión urbana y de los actores políticos: alianzas, riesgos y arenas. Alianzas transversales, reconfiguración de la política y desarrollo urbano: escenarios del presente y del futuro". En: TORRES RIBEIRO A. C. (comp.). El rostro urbano de América Latina. CLACSO Consejo Latinoamericano de Ciencias Sociales.

RIBERA MASGRAU, L. (2004). Los Mapas de Riesgo de Inundaciones: representación de la vulnerabilidad y aportaciones de las innovaciones tecnológicas. Documents d'Anàlisi Geogràfica. Barcelona, n. 43, pp. 153-171.

ROTGER, D. V. y SANZ RESSEL, K. (2016). Paisaje y comunicación social en la gestión del riesgo hídrico. El caso del Gran La Plata. Revista Proyección. Mendoza, v. 5, n. 22, pp. 132-152.

SECRETARIA DE AMBIENTE Y DESARROLLO SUSTENTABLE DE LA NACIÓN (2015). Inundaciones urbanas y cambio climático. Recomendaciones para la gestión. Disponible en: http://ambiente.gob.ar/ wp-content/uploads/Cambio-Climatico_-web.pdf. Acceso en: 10 diciembre 2017.

Texto recebido em 18/fev/2018 Texto aprovado em 20/abr/2018 